\title{
DISTRIBUIÇÃO DO SISTEMA RADICULAR DE UMA CULTURA DE AVEIA FORRAGEIRA ${ }^{1}$
}

\author{
Lorival Fante Jr. ${ }^{2,6 *}$; Klaus Reichardt ${ }^{2,3,5}$; Lucio André de Castro Jorge ${ }^{4}$; Osny Oliveira Santos \\ Bacchi $^{2,5}$ \\ ${ }^{2}$ Laboratórios de Física e Biogeoquímica do Solo - CENA/USP, C.P. 96 - CEP: 134418-970 - Piracicaba, SP. \\ ${ }^{3}$ Depto. de Ciências Exatas - ESALQ/USP, C.P. 9 - CEP: 13418-970 - Piracicaba, SP. \\ ${ }^{4}$ Embrapa Instrumentação Agropecuária, C.P. 741 - CEP: 13560-970 - São Carlos, SP. \\ ${ }^{5}$ Bolsista do CNPq. \\ ${ }^{6}$ Bolsista da FAPESP. \\ *e-mail: I.fante@merconet.com.br
}

RESUMO: Avaliou-se a distribuição das raízes de uma aveia forrageira através da aplicação dos métodos do anel volumétrico, da placa com pregos, do trado e do auxiliado por processamento de imagens. A aveia forrageira foi cultivada em latossolo vermelho-escuro, sob dois tratamentos distintos em função da manutenção da capacidade de água disponível no solo (CAD): tratamento 1 - $50 \%$ da CAD; tratamento $2-80 \%$ da CAD. Foram realizadas 3 amostragens, aos 25, 60 e 115 dias após a emergência. As raízes da aveia forrageira concentraram-se nas camadas iniciais do solo, cerca de $90 \%$ entre 0 a $20 \mathrm{~cm}$. Os resultados encontrados para a quantificação das raízes variaram com o tratamento, a metodologia e a época de amostragem. Verificou-se a existência de correlações entre os resultados determinados a partir das grandezas massa seca de raiz e presença radicular. A variabilidade da distribuição das raízes no solo foi dependente do método de avaliação utilizado. Os menores valores dos coeficientes de variação foram observados para o método do trado, indicando que a menor variabilidade da distribuição das raízes tem maior relação com o volume da amostra do que com o número de pontos amostrados.

Palavras-chave: aveia forrageira, sistema radicular, processamento de imagem, água do solo

\section{ROOT SYSTEM DISTRIBUTION OF A FORRAGE OAT CROP}

ABSTRACT: Forage oat root distribution was evaluated through the application of the methods of the volumetric ring, of the nail plate, of the auger, and of image processing. The crop was grown on a Dark Red Latosol, submitted to two different treatments in relation to water avalilability: 1. Mantaining at least $50 \%$ of the soil available water, and 2. Mantaining $80 \%$. Three samplings were perfoemed, 25, 60 , and 115 days after emergence. Oat roots were concentrated in the top soil layer, about $90 \%$ from 0 to 20 cm depth. Results of root quntification show, in general, distinct with respect to treatment, methodology and sampling date. Correlations were found between data of dry root weigth and root presence. The root variability in the soil depended on the method of evaluation. The lowest coefficients of variation were found for the auger method, indicating that the lowest variability of root distribution has a greater relation to sample volume then on the number of sampling points.

Key words: forage oat, root system, image processing, soil water

\section{INTRODUÇÃO}

A avaliação do sistema radicular de uma cultura pode ser considerado como fundamental no diagnóstico de sistemas de manejo que visam a otimização da produtividade agrícola. A distribuição das raízes no solo é resultante de uma série de processos complexos e dinâmicos, que incluem as interações entre o ambiente, o solo e as plantas em pleno crescimento. Em linhas gerais, segundo Bohm
(1979), estudos sobre crescimento radicular devem ser feitos a partir da avaliação das características das raízes, como massa, comprimento e área, no tempo e no espaço, em conjunto com os fatores que influenciam a distribuição do sistema radicular, como densidade e porosidade do solo, água e ar disponíveis no solo, nutrientes e $\mathrm{pH}$, dentre outros.

Em estudos sobre as raízes e suas interações com o solo (interface solo-raiz), a metodologia de quantificação destaca-se como principal fator limitante. Segundo Schuurmann \&

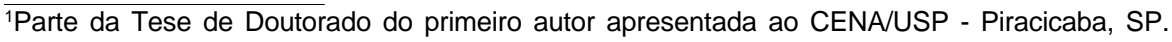


Goedewaagen (1971), Bohm (1979) e Kopke (1981), existem vários métodos que permitem investigar a distribuição do sistema radicular, entretanto, a avaliação das raízes em termos de volume explorado, massa seca e comprimento radicular é tarefa difícil e grandes limitações são encontradas em qualquer técnica, como o tempo gasto, a pouca informação obtida e a variabilidade dos resultados.

Dependendo dos objetivos de estudo, uma avaliação adequada da distribuição das raízes no solo, em função de diferentes variáveis de quantificação, pode exigir a aplicação de metodologias distintas, fato este que pode levar a resultados discrepantes, com significativa variabilidade nos resultados, como foi observado por Kopke (1981) na avaliação do sistema radicular da aveia por diferentes metodologias. Analisando resultados sobre a distribuição das raízes obtidos por diferentes métodos, Kücke et al. (1995), verificaram que eles não podem ser comparados diretamente, exigindo fatores de correlação entre os respectivos resultados, fatores que variaram com a cultura em questão, com o tipo de solo e com a época de amostragem. No trabalho também foi verificado, em função do esquema de amostragem e do tamanho da amostra, altos valores para os coeficientes de variação, indicando significativo efeito da variabilidade espacial sobre a distribuição das raízes no solo.

Revisando diversos trabalhos sobre interações entre raiz e solo, Smucker \& Aiken (1992), Bathke et al. (1992) e Longsdon \& Linden (1992) destacaram a necessidade de se ampliar a utilização de técnicas auxiliadas por processamento de imagens e/ou desenvolvimento de novas técnicas, a fim de possibilitar uma melhor compreensão da interface solo-raiz, bem como contribuir para o desenvolvimento de modelos mais complexos sobre a distribuição do sistema radicular (Diggle, 1988), incluindo análises bi e tridimensionais dessa distribuição e, consequentemente, questões sobre sua variabilidade espacial e temporal.

As metodologias que utilizam técnicas auxiliadas por imagens para avaliar o sistema radicular (Tardieu \& Manichon, 1986; Zoon \& Vantienteren, 1990; Pan \& Bolton, 1991), bem como a aplicação de métodos não destrutivos, como a utilização do minirhizotron (Ferguson \& Smucker, 1989; Rush et al., 1990; Cheng et al., 1991), tem se mostrado como poderosas ferramentas. No Brasil, o desenvolvimento e a utilização destas metodologias estão se concretizando. Crestana et al. (1994) e Jorge et al. (1994) apresentaram novas metodologias auxiliares para o estudo do sistema radicular através da técnica de processamento de imagens, metodologias que também tem se apresentado como relevantes para estes estudos.

Neste sentido, considerando-se a importância da utilização de metodologias auxiliadas por processamento de imagens digitais na avaliação das raízes e as exigências quanto a sua aplicabilidade em comparação aos métodos de avaliação convencionais, e considerando-se que os resultados para a quantificação do sistema radicular dependem da metodologia empregada, o presente trabalho tem como objetivos: 1- utilizar a metodologia auxiliada por imagens na avaliação das raízes e verificar sua aplicabilidade em comparação com outras metodologias já existentes; 2 - avaliar o sistema radicular em termos de diferentes características da raiz, identificando as correlações existentes entre estas em função das metodologias aplicadas; 3 - verificar os efeitos da variabilidade espacial da distribuição das raízes no solo.

\section{MATERIAL E MÉTODOS}

A cultura avaliada foi a aveia forrageira, variedade São Carlos, semeada em latossolo vermelho-escuro de classe textural argila, do Município de São Carlos - SP, e cultivada sob dois tratamentos distintos quanto a capacidade de água disponível no solo (CAD), em duas parcelas de $20 \times 100 \mathrm{~cm}$, com espaçamento de $20 \mathrm{~cm}$ entre linhas e 300 sementes por $\mathrm{m}^{2}$, sendo: tratamento $1=50 \%$ da $C A D$; tratamento $2=80 \%$ da CAD. O balanço hídrico da água no solo foi monitorado durante o ciclo da cultura, utilizando-se tensiômetros a diferentes profundidades e sonda de nêutrons (Bacchi et al., 1996).

As amostragens do sistema radicular foram realizadas em três épocas distintas em função dos dias após a emergência das plantas (DAE): $1^{\mathrm{a}}$ amostragem - entre 25 e $30 \mathrm{DAE} ; 2^{\mathrm{a}}$ amostragem - entre 60 e $65 \mathrm{DAE} ; 3^{\mathrm{a}}$ amostragem - entre 110 e 115 DAE. Nessas amostragens foram empregados dois métodos para a quantificação das raízes, um usando processamento de imagens (Crestana et al., 1994) e outro anel volumétrico. $\mathrm{Na}$ última amostragem utilizou-se mais dois métodos de avaliação, o da placa com pregos e o do trado (Schuurmann \& Goedwaagen, 1971; Bohm, 1979).

Em paredes verticais de trincheiras de 
solo, de $100 \mathrm{~cm}$ de largura e $50 \mathrm{~cm}$ de profundidade foram moldados perfis, paralelamente à linha da cultura e a uma distância de aproximadamente $3 \mathrm{~cm}$ desta. Procedeu-se a lavagem de cada perfil com jato d'água para a remoção de uma fina camada de solo, em torno de $5 \mathrm{~mm}$, visando promover uma melhor exposição das raízes. Para cada perfil obtiveram-se 50 imagens em regiões de $10 \times 10$ $\mathrm{cm}$ usando filmadora de vídeo comum, imagens que foram digitalizadas utilizando-se placa digitalizadora de resolução espacial de 512 x 512 "pixels" e 256 tons de cinza por "pixel". O processamento das imagens digitais foi feito pelo "software" SIARCS (Sistema Integrado para Análise de Raízes e Cobertura do Solo), desenvolvido pela EMBRAPA/ CNPDIA de São Carlos - SP (Crestana et al., 1994), em conjunto com outras ferramentas computacionais que processam imagens e permitem melhorar a qualidade de resolução das mesmas, como o COREL DRAW. A quantificação das raízes por esta metodologia foi feita pela razão entre o número de "pixels" selecionados na imagem e correspondentes às raízes e o número total de "pixels" da mesma imagem, grandeza denominada de densidade radicular por presença de raiz (DRPR), cuja unidade é expressa em porcentagem (Fante Júnior, 1997).

Após a filmagem de cada perfil do solo retirou-se, por região de $10 \times 10 \mathrm{~cm}$, uma amostra indeformada de solo via anel volumétrico, com volume aproximado de $50 \mathrm{~cm}^{3}$ (diâmetro $\cong 4,6 \mathrm{~cm}$ e altura $\cong 3,0 \mathrm{~cm}$ ). As raízes foram separadas do solo por dispersão em água e utilização de peneira de $0,2 \mathrm{~mm}$, lavadas em água para a completa remoção do solo e colocadas em estufa a $60^{\circ} \mathrm{C}$ por 24 horas para a obtenção da massa seca radicular.

Em um perfil de solo moldado como especificado anteriormente, em outra linha da cultura, distando aproximadamente $100 \mathrm{~cm}$ da área de amostragem com anel volumétrico e auxiliado por imagens, foi cravada, a partir da superfície, uma placa de madeira com pregos de $10 \mathrm{~cm}$ de comprimento, de $50 \mathrm{~cm}$ de largura por $50 \mathrm{~cm}$ de profundidade. Os pregos foram atravessados na placa com espaçamento uniforme, formando uma malha de $5 \times 5 \mathrm{~cm}$. Depois de retirado do perfil do solo, o sistema placa com pregos foi lavado com água para retirar o solo com um mínimo de perdas de raízes, sendo estas posteriormente retiradas do sistema em camadas de $10 \mathrm{~cm}$, lavadas em água para remover todo o solo e colocadas em estufa a $60^{\circ} \mathrm{C}$ por 24 horas para a obtenção da massa seca radicular.

Utilizando-se de um trado manual de caneca de $5 \mathrm{~cm}$ de diâmetro por $10 \mathrm{~cm}$ de altura, foram retiradas, da superfície até $50 \mathrm{~cm}$ de profundidade, três amostras deformadas de solo por camada de $10 \mathrm{~cm}$, em locais separados entre si por aproximadamente $50 \mathrm{~cm}$, ao longo de uma linha paralela à da cultura e distando em torno de $3 \mathrm{~cm}$ desta. A linha da cultura amostrada através do trado foi a mesma considerada para o método da placa com pregos, com distância de $300 \mathrm{~cm}$ entre os dois locais de amostragem. A separação das raízes das amostras de solo e a secagem foram realizadas como especificado para o método do anel volumétrico.

A quantificação das raízes pelos métodos do anel volumétrico, placa com pregos e trado foi feita pela razão entre a massa seca radicular e o volume de solo amostrado, grandeza denominada de densidade radicular por massa (DRM).

\section{RESULTADOS E DISCUSSÃO}

Os resultados obtidos da quantificação do sistema radicular da aveia forrageira, em função da amostragem realizada, da camada de solo avaliada, da metodologia empregada e para os dois tratamentos considerados, estão apresentados nas TABELAS 1 e 2 . Os valores são médias referentes à $\mathrm{N}$ amostras obtidas por camada de solo com seus respectivos coeficientes de variação $(\mathrm{CV})$, sendo $\mathrm{N}=10$ para os métodos auxiliados por imagens e do anel volumétrico, $\mathrm{N}=3$ para o método do trado e $\mathrm{N}=1$ para 0 método da placa com pregos; para este último método os valores de CV não são determináveis.

Pode-se observar nas TABELAS 1 e 2 que, independentemente do método aplicado, do tratamento e da época de amostragem, as raízes estão concentradas nas camadas iniciais, ou seja, 0-20 cm. Na TABELA 3 são apresentados os resultados médios acumulados nas camadas de solo de $0-20 \mathrm{~cm}$ e $20-50 \mathrm{~cm}$, e os respectivos percentuais encontrados em função do total raízes acumulado no perfil do solo amostrado. Verifica-se, desta forma, que mais de $80 \%$ das raízes estão distribuídas na camada de $0-20 \mathrm{~cm}$, entre $83 \%$ a $96 \%$, dependendo do tratamento, da amostragem e da metodologia de avaliação. Em estudos sobre a distribuição do sistema radicular 
TABELA 1 - Quantificação das raízes de aveia forrageira pelos métodos de extração, em camadas de $10 \mathrm{~cm}$ de solo, para os dois tratamentos e para as três amostragens. As médias de densidade radicular por massa (DRM) e os coeficientes de variação (CV) correspondem a $\mathrm{N}$ amostras retiradas por camada.

\begin{tabular}{|c|c|c|c|c|c|}
\hline amostragem & camada $(\mathrm{cm})$ & $\mathrm{DRM}\left(\mathrm{kg} \mathrm{m}^{-3}\right)$ & CV (\%) & $\mathrm{DRM}\left(\mathrm{kg} \mathrm{m}^{-3}\right)$ & $\mathrm{CV}(\%)$ \\
\hline & & \multicolumn{2}{|c|}{ Tratamento 1} & \multicolumn{2}{|c|}{ Tratamento 2} \\
\hline \multirow{6}{*}{$1^{\mathrm{a}}$} & $=\quad 010$ & 0550 & 181 & $1 c 50$ & \\
\hline & 1020 & 0708 & 553 & 1000 & 171 \\
\hline & $10-20$ & 0,708 & 55,3 & 1,009 & 47,1 \\
\hline & $20-30$ & 0,115 & 59,2 & 0,129 & 73,2 \\
\hline & $30-40$ & 0,079 & 92,5 & 0,087 & 67,7 \\
\hline & $40-50$ & 0,067 & 48,8 & 0,061 & 40,6 \\
\hline \multirow[t]{5}{*}{$2^{\mathrm{a}}$} & $0-10$ & 3,077 & 39,9 & 2,754 & 43,7 \\
\hline & $10-20$ & 0,377 & 30,6 & 0,753 & 39,7 \\
\hline & $20-30$ & 0,237 & 76,8 & 0,066 & 51,9 \\
\hline & $30-40$ & 0,167 & 75,6 & 0,061 & 41,3 \\
\hline & $40-50$ & 0,237 & 63,5 & 0,107 & 42,4 \\
\hline \multirow[t]{5}{*}{$3^{\mathrm{a}}$} & $0-10$ & 3,753 & 26,9 & 2,535 & 42,8 \\
\hline & $10-20$ & 0,464 & 92,3 & 0,73 & 67,4 \\
\hline & $20-30$ & 0,144 & 48,7 & 0,119 & 53,9 \\
\hline & $30-40$ & 0,192 & 27,8 & 0,103 & 56,6 \\
\hline & $40-50$ & 0,129 & 41,5 & 0,13 & 85,3 \\
\hline \multicolumn{6}{|c|}{ placa com pregos $(\mathrm{N}=1)$} \\
\hline \multirow[t]{5}{*}{$3^{\mathrm{a}}$} & $0-10$ & 2,532 & * & 0,98 & * \\
\hline & $10-20$ & 0,409 & * & 1,071 & * \\
\hline & $20-30$ & 0,126 & * & 0,279 & * \\
\hline & $30-40$ & 0,177 & * & 0,044 & * \\
\hline & $40-50$ & 0,257 & * & 0,029 & * \\
\hline \multicolumn{6}{|l|}{ trado $(\mathrm{N}=3)$} \\
\hline \multirow[t]{5}{*}{$3^{\mathrm{a}}$} & $0-10$ & 2,486 & 9,3 & 2,598 & 9,4 \\
\hline & $10-20$ & 1,729 & 50,9 & 2,287 & 9,4 \\
\hline & $20-30$ & 0,588 & 62,5 & 0,455 & 36,5 \\
\hline & $30-40$ & 0,345 & 27,9 & 0,131 & 33,1 \\
\hline & $40-50$ & 0,345 & 25,2 & 0,08 & 13,3 \\
\hline
\end{tabular}

$\left(^{*}\right)$ valores de CV não determináveis, $\mathrm{N}=1$.

de quatro culturas agrícolas em função do manejo do solo, Merten \& Mielniczuk (1991), verificaram que em torno de $90 \%$ das raízes da aveia preta concentraram-se nas camadas superficiais $(0-20$ $\mathrm{cm})$.

Os altos valores encontrados para CV, indicam considerável variabilidade espacial da distribuição das raízes no perfil do solo. Esses resultados também sugerem que os efeitos desta variabilidade tem maior relação com o tamanho da amostra do que com o número de amostras considerado, haja visto a comparação entre os 
TABELA 2 - Quantificação das raízes de aveia forrageira pelo método auxiliado por processamento de imagens, em camadas de $10 \mathrm{~cm}$ de solo, para os dois tratamentos e para as três amostragens. As médias de densidade radicular por presença de raiz (DRPR) e os coeficientes de variação (CV) correspondem a 10 amostras retiradas por camada.

\begin{tabular}{|c|c|c|c|c|c|}
\hline amostragem & $\begin{array}{c}\text { camada } \\
(\mathrm{cm})\end{array}$ & $\begin{array}{c}\text { DRPR } \\
(\%)\end{array}$ & $\begin{array}{l}\text { CV } \\
(\%)\end{array}$ & $\begin{array}{c}\text { DRPR } \\
(\%)\end{array}$ & $\begin{array}{l}\text { CV } \\
(\%)\end{array}$ \\
\hline & & \multicolumn{2}{|c|}{ tratamento 1} & \multicolumn{2}{|c|}{ tratamento 2} \\
\hline \multicolumn{6}{|c|}{ processamento de imagens $(\mathrm{N}=10)$} \\
\hline \multirow[t]{5}{*}{$1^{\mathrm{a}}$} & $0-10$ & 5,0 & 8,7 & 4,9 & 33,3 \\
\hline & $10-20$ & 2,1 & 52,6 & 2,5 & 47,8 \\
\hline & $20-30$ & 0,7 & 83,3 & 0,6 & 60,0 \\
\hline & $30-40$ & 0,3 & 100,0 & 0,1 & 100,0 \\
\hline & $40-50$ & 0,6 & 60,0 & 0,4 & 75,0 \\
\hline \multirow[t]{5}{*}{$2^{\mathrm{a}}$} & $0-10$ & 3,4 & 29,0 & 4,0 & 40,0 \\
\hline & $10-20$ & 0,7 & 66,7 & 0,8 & 66,7 \\
\hline & $20-30$ & 0,4 & 50,0 & 0,3 & 100,0 \\
\hline & $30-40$ & 0,1 & 100,0 & 0,2 & 50,0 \\
\hline & $40-50$ & 0,4 & 75,0 & 0,6 & 80,0 \\
\hline \multirow[t]{5}{*}{$3^{a}$} & $0-10$ & 4,0 & 41,7 & 3,7 & 47,1 \\
\hline & $10-20$ & 0,8 & 100,0 & 1,7 & 100,0 \\
\hline & $20-30$ & 0,3 & 33,3 & 0,3 & 66,7 \\
\hline & $30-40$ & 0,4 & 75,0 & 0,4 & 75,0 \\
\hline & $40-50$ & 0,4 & 25,0 & 0,3 & 33,3 \\
\hline
\end{tabular}

valores de CV obtidos para os métodos do trado, maior volume amostrado e menor número de amostras, e do anel volumétrico e auxiliado por processamento de imagens, menor volume amostrado e maior número de amostras. Em média, o valor de CV obtido para o método do trado apresentou-se 2 vezes menor daqueles encontrados para os demais métodos, sendo que o tamanho da região amostrada pela aplicação do método do trado foi de aproximadamente 4 vezes maior em relação aos outros métodos.

O esquema experimental e os objetivos centrais deste trabalho, bem como as dificuldades de amostragem e experimentais em estudos sobre avaliação de raízes, não permitiram que os resultados fossem discriminados através de teste de médias. Entretanto, independente de uma avaliação estatística convencional e apesar dos elevados valores encontrados para CV, os resultados obtidos sugerem fortes indicativos quanto à verificação de diferenças e concordâncias entre estes.

Com relação aos métodos que consideraram a DRM para a quantificação das raízes, pode-se verificar na camada de solo de 10-20 cm, que o tratamento 2 apresentou maior concentração radicular quando comparado ao tratamento 1 , isto independentemente do método de avaliação e da amostragem. Por outro lado, para a camada de $0-10 \mathrm{~cm}$, com exceção à aplicação do método do trado, verifica-se uma maior concentração radicular para o tratamento 1 em comparação ao tratamento 2. Nas demais camadas de solo amostradas, no geral, verificouse maior concentração de massa radicular no tratamento 1 do que no 2, independentemente dos métodos de extração empregados (TABELA 1). Este fato também foi observado para os valores médios de DRM acumulados nas camadas $0-20 \mathrm{~cm}$ e $20-50 \mathrm{~cm}$ (TABELA $3 \mathrm{a}$ ).

Desta forma, os resultados obtidos para 
TABELA 3 - Quantificação das raízes de aveia forrageira pelos métodos empregados, para as camadas de solo de $0-20 \mathrm{~cm}$ e $20-50 \mathrm{~cm}$, para os dois tratamentos e as três amostragens. Os valores de densidades radiculares por massa (DRM) e por presença de raiz (DRPR) são médias por camada considerada e os respectivos percentuais são em relação ao total acumulado no perfil do solo.

\begin{tabular}{lccccc}
\hline (a) métodos de extração & & & \\
& camada & DRM & DRM \\
amostragem & $(\mathrm{cm})$ & $\left(\mathrm{kg} \cdot \mathrm{m}^{-3}\right)$ & $(\%)$ & $\left(\mathrm{kg} \cdot \mathrm{m}^{-3}\right)$ & $(\%)$ \\
\hline
\end{tabular}

anel volumétrico

\begin{tabular}{|c|c|c|c|c|c|}
\hline \multirow[t]{2}{*}{$1^{\mathrm{a}}$} & $0-20$ & 1,633 & 95 & 1,333 & 94 \\
\hline & $20-50$ & 0,087 & 5 & 0,092 & 6 \\
\hline \multirow[t]{2}{*}{$2^{\mathrm{a}}$} & $0-20$ & 1,727 & 89 & 1,754 & 96 \\
\hline & $20-50$ & 0,214 & 11 & 0,078 & 4 \\
\hline \multirow[t]{2}{*}{$3^{a}$} & $0-20$ & 2,109 & 93 & 1,633 & 93 \\
\hline & $20-50$ & 0,155 & 7 & 0,117 & 7 \\
\hline \multicolumn{6}{|c|}{ placa com pregos } \\
\hline \multirow[t]{2}{*}{$3^{a}$} & $0-20$ & 1,472 & 88 & 1,026 & 89 \\
\hline & $20-50$ & 0,187 & 12 & 0,117 & 11 \\
\hline \multicolumn{6}{|l|}{ trado } \\
\hline \multirow[t]{2}{*}{$3^{a}$} & $0-20$ & 2,108 & 83 & 2,443 & 92 \\
\hline & $20-50$ & 0,426 & 17 & 0,222 & 8 \\
\hline \multicolumn{6}{|c|}{ (b) método auxiliado por processamento de imagens } \\
\hline \multirow[t]{3}{*}{ amostragem } & camada & DRPR & & DRPR & \\
\hline & $(\mathrm{cm})$ & $(\%)$ & $(\%)$ & $(\%)$ & (\%) \\
\hline & & \multicolumn{2}{|c|}{ tratamento 1} & \multicolumn{2}{|c|}{ tratamento 2} \\
\hline \multirow[t]{2}{*}{$1^{\mathrm{a}}$} & $0-20$ & 3,6 & 86 & 3,7 & 90 \\
\hline & $20-50$ & 0,6 & 14 & 0,4 & 10 \\
\hline \multirow[t]{2}{*}{$2^{\mathrm{a}}$} & $0-20$ & 2,1 & 88 & 2,4 & 86 \\
\hline & $20-50$ & 0,3 & 12 & 0,4 & 14 \\
\hline \multirow[t]{2}{*}{$3^{a}$} & $0-20$ & 2,4 & 86 & 2,7 & 90 \\
\hline & $20-50$ & 0,4 & 14 & 0,3 & 10 \\
\hline
\end{tabular}

DRM pela aplicação dos métodos de extração sugerem, de maneira geral, uma concordância e coerência qualitativas, indicando uma maior quantidade e melhor distribuição de massa radicular para o tratamento 1 em relação ao tratamento 2, apesar de maior capacidade de água disponível no solo neste último tratamento.

Entretanto, na $3^{\mathrm{a}}$ amostragem, onde os três métodos de extração foram empregados, os resultados de DRM (TABELAS 1 e $3 a$ ), em sua maioria, apresentaram diferenças e discrepâncias quantitativas, sendo que algumas mostraram-se relevantes. Na camada de solo de $0-10 \mathrm{~cm}$ e no tratamento 1 , o método do anel volumétrico superestimou o valor de DRM em relação aos outros dois métodos utilizados, enquanto no tratamento 2 o método da placa com pregos subestimou a avaliação de DRM. Essas discrepâncias também são verificadas na camada de solo de $10-20 \mathrm{~cm}$, com o método do trado apresentando valores superiores aos demais métodos de extração, e estes apresentando 
diferenças entre si considerando-se os tratamentos 1 e 2. Indicações como as mencionadas, são verificadas também na camada de $20-30 \mathrm{~cm}$. Considerando-se os valores médios de DRM, nas camadas de solo de $0-20 \mathrm{~cm}$ e $20-50 \mathrm{~cm}$, diferenças quantitativas entre os métodos de avaliação também são observadas, conforme se verifica na TABELA 3a.

Estas diferenças e discrepâncias verificadas para os resultados encontrados pela aplicação dos métodos de extração e referentes à $3^{a}$ amostragem, estão associadas não somente ao método utilizado na quantificação das raízes, mas também à variabilidade espacial da distribuição do sistema radicular, uma vez que os valores dos CV foram altos e os locais de amostragem foram distintos para os métodos empregados. Tal aspecto pode ser considerado como um fator limitador no emprego de métodos destrutivos em estudos sobre avaliação de raízes, principalmente quando existe a necessidade de amostragens ao longo do tempo de desenvolvimento de uma cultura agrícola e, por esta razão, as amostragens não são possíveis de serem realizadas no mesmo local.
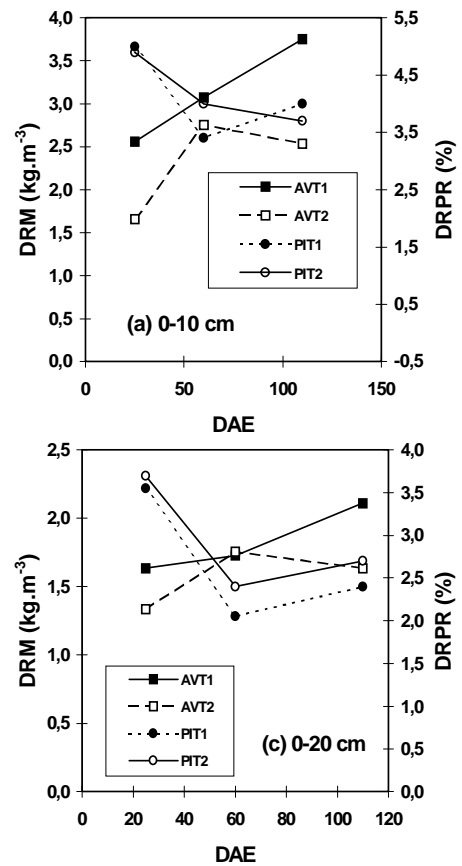

Analisando-se os resultados obtidos pelo método auxiliado por processamento de imagens, cuja grandeza utilizada na quantificação das raízes foi a DRPR (TABELAS 2 e 3b), estes mostraramse, no geral, conflitantes quando comparados aos do método do anel volumétrico, cuja grandeza utilizada foi DRM (TABELAS 1 e 3a).

A aplicação do método auxiliado por processamento de imagens, ao contrário do que ocorreu com os valores de DRM para os métodos de extração, não proporcionou resultados de tal forma a sugerir diferenças entre os tratamentos 1 e 2, com exceção à $3^{a}$ amostragem, onde também verificou-se na camada de solo de 10$20 \mathrm{~cm}$ uma maior presença radicular para o tratamento 2 quando comparado ao tratamento 1 , e à $1^{\text {a }}$ amostragem, onde foi observado um maior valor médio acumulado de DRPR na camada de solo de $20-50 \mathrm{~cm}$ para o tratamento 1 em comparação ao tratamento 2 .

Os resultados obtidos para DRM e DRPR, em função da época de amostragem, em sua maioria, apresentaram comportamentos distintos, como pode ser observado nas respectivas tabelas, bem como na Figura 1.
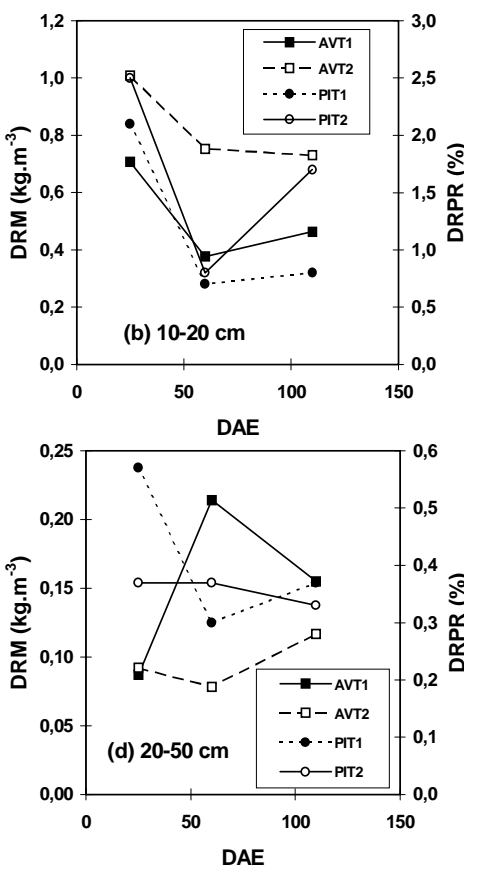

Figura 1 - Comportamento dos valores de DRM e DRPR em função da época de amostragem (DAE), para os dois tratamentos avaliados e para as camadas de solo: (a) 0-10 cm, (b) 10-20 cm, (c) 0-20 cm e (d) 20-50 cm, sendo: AVT1 - anel volumétrico tratamento 1, AVT2 - anel volumétrico tratamento 2, PIT1 - processamento de imagens tratamento 1 e PIT2 - processamento de imagens tratamento 2 . 
Para a camada de solo de $0-10 \mathrm{~cm}$ os resultados para DRM indicam um aumento da massa radicular em relação aos DAE, isto para os dois tratamentos avaliados. Contudo, os resultados para DRPR em função dos DAE sugerem uma diminuição da presença de raízes nesta camada de solo. Tais comportamentos podem também ser observados para as camadas de solo de $0-20 \mathrm{~cm}$ e 20-50 $\mathrm{cm}$. Quanto à camada de solo de 10-20 $\mathrm{cm}$, os resultados para DRM e DRPR apresentaram comportamentos similares, verificando-se um indicativo de diminuição da massa e da presença radiculares em função dos DAE.

As diferenças encontradas entre as quantificações determinadas pela massa radicular e pela presença de raízes, em princípio, podem ser entendidas com base nas avaliações feitas pelas grandezas distintas, DRM e DRPR, ou seja, a determinação de uma considerável massa radicular, em uma dada região amostrada, não implica que nesta região existe uma grande presença de raízes, isto porque a relação entre tais grandezas depende, dentre outros fatores, da dimensão das raízes. Entretanto, para a cultura avaliada, verificou-se que a maior parte das raízes apresentou pequenas variações de diâmetro ao longo do perfil do solo, diâmetro este determinado pelo processamento das imagens digitais através do programa SIARCS (Fante Júnior, 1997). Por outro lado, a quantificação das raízes a partir da sua massa não implica, necessariamente, ser idêntica à quantificação radicular dada pela presença de raiz, mas sim que essas grandezas podem estar correlacionadas entre si. Assim, determinaram-se correlações entre DRM e DRPR, a partir dos resultados médios obtidos por camada de solo de $10 \mathrm{~cm}$ e considerando-se: cada amostragem e cada tratamento; as três amostragens para cada tratamento; as três amostragens e os dois tratamentos.

Os resultados encontrados destes ajustes estão apresentados na TABELA 4 e alguns também mostrados na Figura 2. Verificou-se que os ajustes foram satisfatórios e que as melhores correlações entre DRM e DRPR foram obtidas para cada amostragem e cada tratamento, com as constantes dos ajustes variando em relação à amostragem e ao tratamento, indicando um aumento do coeficiente angular em função do tempo de desenvolvimento da aveia forrageira e maiores valores das constantes para o tratamento 1 em comparação ao tratamento 2 .

TABELA 4 - Resultados obtidos para os ajustes dos comportamentos entre as grandezas DRM (densida de radicular por massa) e DRPR (densidade radicular por presença de raiz), a partir de funções lineares: $D R M=a D R P R+b$, sendo $a$ e $b$, respectivamente, os coeficientes angular $e$ linear da função e $R^{2}$ o respectivo coeficiente de determinação.

(a) em função do tratamento e da amostragem

tratamento 1

tratamento 2

\begin{tabular}{lcccccc} 
amostragem & $1^{\mathrm{a}}$ & $2^{\mathrm{a}}$ & $3^{\mathrm{a}}$ & $1^{\mathrm{a}}$ & $2^{\mathrm{a}}$ & $3^{\mathrm{a}}$ \\
$\mathrm{a}\left(\mathrm{kg} \cdot \mathrm{m}^{-3}\right)$ & 0,545 & 0,926 & 0,994 & 0,352 & 0,718 & 0,700 \\
$\mathrm{~b}\left(\mathrm{~kg} \cdot \mathrm{m}^{-3}\right)$ & $-0,242$ & $-0,107$ & $-0,237$ & $-0,009$ & $-0,099$ & $-0,172$ \\
$\mathrm{R}^{2}$ & 0,985 & 0,990 & 0,998 & 0,982 & 0,976 & 0,975 \\
\hline
\end{tabular}

(b) em função do tratamento, considerando-se as três amostragens tratamento 1

tratamento 2

\begin{tabular}{lrr}
$\mathrm{a}\left(\mathrm{kg} \cdot \mathrm{m}^{-3}\right)$ & 0,727 & 0,526 \\
$\mathrm{~b}\left(\mathrm{~kg} \cdot \mathrm{m}^{-3}\right)$ & $-0,129$ & $-0,042$ \\
$\mathrm{R}^{2}$ & 0,855 & 0,836 \\
\hline
\end{tabular}

(c) considerando-se os dois tratamentos e as três amostragens

\begin{tabular}{lc}
$\mathrm{a}\left(\mathrm{kg} \cdot \mathrm{m}^{-3}\right)$ & 0,622 \\
$\mathrm{~b}\left(\mathrm{~kg} \cdot \mathrm{m}^{-3}\right)$ & $-0,084$ \\
$\mathrm{R}^{2}$ & 0,82 \\
\hline
\end{tabular}



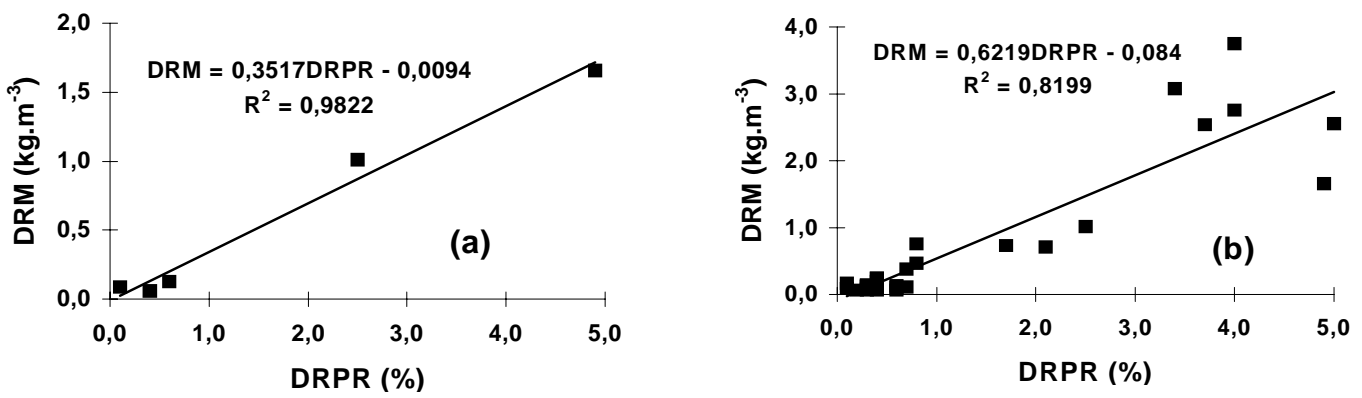

Figura 2 - Correlação entre as grandezas DRM, obtida pelo anel volumétrico, e DRPR, obtida por processamento de imagens. Os valores são médias referentes a 10 repetições por camada de solo de $10 \mathrm{~cm}$, sendo: (a) tratamento 2 e 1a amostragem e (b) as três amostragens e os dois tratamentos.

\section{CONCLUSÕES}

Com base nos apontamentos feitos na discussão dos resultados obtidos para a quantificação das raízes da cultura de aveia forrageira em questão, pode-se delinear alguns indicativos como conclusões do presente trabalho, ou seja:

- em torno de $90 \%$ das raízes concentraram-se na camada do solo de $0-20 \mathrm{~cm}$;

- a distribuição do sistema radicular no solo apresentou considerável variabilidade espacial, variabilidade esta que mostrou maior relação com o tamanho das amostras do que com o número de amostras;

- a aplicação de métodos destrutivos na quantificação de raízes ao longo do tempo, apresentou um fator limitador intrínseco, relacionado à impossibilidade de amostragens na mesma região do solo e aos efeitos da variabilidade espacial do sistema radicular;

- as diferenças e discrepâncias quantitativas encontradas nos resultados derivados da aplicação dos métodos de extração não se apresentaram de forma sistemática em relação ao método, tratamento e camada de solo amostrada;

- a manutenção da capacidade de água disponível no solo, em $50 \%$ e $80 \%$, não influenciou a distribuição das raízes no solo, registrando-se, no geral, maior massa radicular para o tratamento 1 do que para o tratamento2;

- as grandezas massa radicular e presença radicular, em sua maioria, apresentaram-se conflitantes e com comportamentos distintos em função da época de desenvolvimento da aveia forrageira, sendo que estas grandezas, apesar de distintas, correlacionaram-se linearmente entre si, com melhores ajustes obtidos para cada amostragem e cada tratamento;

- o método auxiliado por processamento de imagens digitais, mostrou-se bastante satisfatório em comparação aos demais métodos empregados, caracterizando-se como uma ferramenta alternativa em estudo sobre raízes.

\section{AGRADECIMENTOS}

Este trabalho recebeu apoio financeiro da FAPESP e foi realizado em parceria entre as instituições de pesquisa: CENA/USP de Piracicaba - SP, EMBRAPA/CNPDIA e EMBRAPA/CPPSE de São Carlos - SP. Agradecemos, desta forma, à FAPESP pelo auxílio à pesquisa e à bolsa concedida durante o desenvolvimento do referido trabalho de doutorado, bem como a todos os pesquisadores, pós-graduandos e técnicos destas instituições que, direta ou indiretamente, contribuíram para a sua concretização, em especial, ao Dr. Silvio Crestana, Dr. Rodolfo Godoy, Dr. Júlio César Martins de Oliveira, Dr. Luiz Henrique Bassoi, José Ademir Rodriques e João Eduardo Pilotto.

\section{REFERÊNCIAS BIBLIOGRÁFICAS}

BACCHI, O.O.S.; GODOY, R.; FANTE JÚNIOR, L.; REICHARDT, K. Balanço hídrico em cultura de aveia forrageira de inverno na região de São Carlos - SP. Scientia Agricola, v.53, n.1, p.172-178, 1996.

BATHKE, G.R.; CASSEL, D.K.; HARGROVE, W.L.; PORTER, P.M. Modification of soil properties and root growth response. Soil Science, v.154, n.4, p.316-329, 1992.

BOHM, W. Methods of studying root systems. New York: Springer-Verlag, 1979. 189p. 
CHENG, W.; COLEMAN, D.S.; BOX JR., J.E. Measuring root turnover using the minirhizotron technique. Agriculture Ecosystems Environment, v.34, n.1/4, p.261-267, 1991.

CRESTANA, S.; GUIMARÃES, M.F.; JORGE, L.A.C.; RALISH, R.; TOZZI, C.L.; TORRE, A.; VAZ, C.M.P. Avaliação da distribuição de raízes no solo auxiliada por processamento de imagens digitais. Revista Brasileira de Ciência do Solo, v.18, n.3, p.365-371, 1994.

DIGGLE, A.J. ROOTMAP: a model in threedimensional coordinates of the growth and structure of fibrous root systems. Plant Soil, v.105, p.169-178, 1988.

FANTE JÚNIOR, L. Sistema radicular da aveia forrageira avaliado por diferentes métodos, incluindo processamento de imagens digitais. Piracicaba, 1997. 119p. Tese (Doutorado) Centro de Energia Nuclear na Agricultura, Universidade de são Paulo.

FERGUSON, J.C.; SMUCKER, A.J.M. Modification of the minirhizotron video camera system for measuring spatial and temporal root dynamics. Soil Science Society of America Journal, v.53,p.1601-1605, 1989.

JORGE, L.A.C.; RESENDE, P.C.S.; POSADAS DURANT, A.; FREITAS JUNIOR, E.; CRESTANA, S. Comparação de técnicas de análise de imagens digitais na determinação do comprimento de raízes. In: REUNIÃO BRASILEIRA DE MANEJO E CONSERVAÇÃO DO SOLO E DA ÁGUA, 10., Florianópolis, 1994. Resumos. Florianópolis: SBCS, 1994. p.314-315.

KOPKE, U. Methods for studying root growth. In: SYMPOSIUM ON THE SOIL/ROOT SYSTEM IN RELATION TO BRAZILIAN AGRICULTURE, Londrina, 1980. Proceedings. Londrina: IAPAR, 1981. p.303-318.
KÜCKE, M.; SCHIMID, H.; SPIESS, A. A comparison of four methods for measuring roots of field crops in three contrasting soils. Plant Soil, v.172, p.63-71, 1995.

LONGSDON, S.D.; LINDEN, D.R. Interactions of earthworms withsoil physical conditions influencing plant growth. Soil Science, v.154, n.4, p.330-337, 1992.

MERTEN, G.H.; MIELNICZUK, J. Distribuição do sistema radicular e dos nutrientes em latossolo roxo sob dois sistemas de preparo de solo. Revista Brasileira de Ciência do Solo, v.15, p.369-374, 1991.

PAN, W.L.; BOLTON, R.P. Root quantification by edge discrimination using a desktop scanner. Agronomy Journal, v.83, n.6, p.1047-1052, 1991.

RUSH, C.M., UPCHURCH, D.R.; GERIK, T.J. In situ observations of Phymatotichum with a borescope minirhizotron system. Phytopathology, v.74, p.104-105, 1990.

SCHUURMANN, J.J.; GOEDEWAAGEN, M.A.J. Methods for the examination of root systems and roots. 2.ed. Wageningen: Pudoc, 1971. $86 \mathrm{p}$.

SMUCKER, A.J.M.; AIKEN, R.M. Dynamic responses to water deficits. Soil Science, v.154, n.4, p.281-289, 1992.

TARDIEU, F.; MANICHON, H. Caracterization en tant que capteur d'etude de la répartition verticale et horizontale des racines. Agronomie, v.6, n.5, p.415-425, 1986.

ZOON, F.C.; VANTIENDEREN, P.H. A rapid quantitative measurement of root length and root branching by microcomputer image analysis. Plant Soil, v.126, p.301-308, 1990.

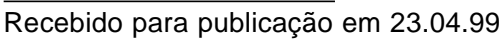

Aceito para publicação em 17.08.99 